\title{
Milices Maï-Maï, Dénonciation De L'impérialisme Et Politisation Des Masses Rurales Au Maniema (RDC)
}

\author{
Fraternel Amuri Misako \\ Professeur au Département de Sciences politiques et administratives \\ Université de Kisangani, RDC \\ doi: 10.19044/esj.2017.v13n17p65 URL:http://dx.doi.org/10.19044/esj.2017.v13n17p65
}

\begin{abstract}
Based on a documentary inquiry aimed at reconstructing the processes of denunciation of imperialism associated with the Congolese Rally for Democracy (RCD)'s war in the Democratic Republic of Congo (DRC), by the maï-maï militia of Maniema, the article examines under the lens of historical criticism (heuristic and hermeneutic) the politicoideological contents of the essential documents whose impact on the rural masses remains crucial: their over-politicization of the latter. The study shows how a political mobilization that initially targeted awareness-raising for the recruitment of new combatants among rural Congolese youths has reactivated the protesting reflex of the rural populations both towards the rebels and their Rwandan allies, and maï-maï combatants. The repeated abuses of the maï-maï militias have thus brought down the nationalist mask which their ideological propaganda maintained until then. The pervasive activism of youths and especially the emergence of radicalized groups confirm the thesis of political violence as an indicator of democratic deficiencies of a weakened state through processes of globalization badly assumed in the African Great Lakes area.
\end{abstract}

Keywords: Democratization, imperialism, Maï-Maï militias, politicization, rural masses of Maniema

\section{Résumé}

Basé sur une enquête documentaire visant à reconstituer les processus de la dénonciation, par les milices maï-maï du Maniema, de l’impérialisme associé avec la guerre du Rassemblement Congolais pour la Démocratie (RCD) en République démocratique du Congo (RDC), l'article examine sous le prisme de la critique historique (heuristique et herméneutique) les contenus politico-idéologiques des documents essentiels dont l'impact sur les masses rurales demeure crucial : la politisation à outrance de ces dernières. 
L'étude montre comment une mobilisation politique qui, au départ, ciblait la sensibilisation aux fins de recrutement de nouveaux combattants parmi les jeunes ruraux congolais, a réactivé le réflexe contestataire des populations rurales à la fois vis-à-vis des rebelles et leurs alliés rwandais, et des combattants maï-maï. Les abus répétés des milices maï-maï ont ainsi fait tomber le masque nationaliste que leur propagande idéologique entretenait jusque-là. L'activisme poussé des jeunes et surtout l'émergence des groupes radicalisés confirment la thèse de la violence politique comme indicateur de carences démocratiques d'un Etat affaibli par des processus de globalisation mal assumés dans la région des Grands Lacs africains.

Mots-clés: Démocratisation, impérialisme, milices maï-maï, masses rurales du Maniema, politisation

\section{Introduction}

«La masse de publications peu fiables et fort médiatisées, déconnectées du contexte historique, hypothèque l'écriture de l'histoire pour l'avenir, tandis que, dans l'immédiat, le public se tourne vers des écrits accessibles et conformes aux vues de son groupe d'appartenance. » (J. Vansina)

La guerre déclenchée le 02 août 1998 par le mouvement politicomilitaire dénommé '“Rassemblement Congolais pour la Démocratie”, RCD en sigle, a bénéficié du soutien des alliés rwandais et ougandais dès ses débuts. La capacité mobilisatrice de ce dernier s'est cependant révélée faible malgré la collaboration de ces mêmes alliés qui avaient permis à l'Alliance des Forces Démocratiques pour la Libération du Congo (AFDL) de prendre le pouvoir à Kinshasa en 1997. Alors que les deux mouvements se réclamaient du nationalisme en vue de la libération de la démocratie confisquée sous le régime de Mobutu, force est de constater que la guerre menée par le RCD contre le régime de Laurent-Désiré Kabila a été jugée inopportune par rapport à celle conduite par l'AFDL (N'Sanda, 2001, 2003a).

Le partage des territoires occupés dits «libérés » entre les forces rwandaises et ougandaises, a donné lieu à l'identification de deux principales zones d'influence. Le Maniema, entre autres provinces de l'Est, revenait ainsi au Rwanda. Ce dernier constituera la cible en fonction de laquelle les milices maï-maï ${ }^{11}$ se comporteront idéologiquement pendant la résistance contre le RCD.

\footnotetext{
${ }^{11}$ Le terme “maï-maï” est bien élucidé dans les pages prochaines.
} 
L'analyse livrée dans cet article s'inscrit dans la perspective des études relatives à l'impact de la privatisation de la violence, cristallisée depuis la transition militariste, sur la politisation des masses rurales par l'élite guerrière issue des milices maï-maï. La question des milices maï-maï abordée ici est consécutive à des réflexions antérieures sur les guerres de libération et leurs effets politico-idéologiques collatéraux. Ces dernières sont riches de leçons en rapport à la fois avec les processus de démocratisation et la persistance des pratiques autoritaires en Afrique (De Villers, 2016).

Tout en s'attribuant une part de rationalité autonome et de légitimité de fait, chaque catégorie d'acteurs présents sur la scène de violences politiques en RDC, affirme sa prééminence de mieux servir le peuple. Pour ce faire, celui-ci est invité à apporter son soutien qui devrait permettre aux acteurs dominants d'atteindre les objectifs d'intérêt général déclarés, notamment garantir la sécurité de tous contre toutes les menaces d'où qu'elles viennent, la justice, l'ordre, la liberté, l'égalité des droits et des obligations, sans oublier le partage équitable des ressources en tant que biens publics. En même temps, la part de légitimité que s'attribue chacune des principales catégories d'acteurs est devenue très contestable dans la mesure où le gouvernement légal incarnant l'Etat formel a perdu le monopole de la violence légitime et n'est plus en mesure d'assurer l'ordre et la sécurité des personnes et de leurs biens; les mouvements politico-militaires luttant pour la libération par la violence abusent à leur tour de la confiance du peuple, même après avoir réussi à se débarrasser du régime en place accusé de dictature. Les milices d'autodéfense populaires, elles qui ne se réclament pas forcément de l'une ou l'autre des deux catégories précédentes, ont aussi leur part d'abus, même quand elles font montre de discipline dans des domaines bien spécifiques.

L'implication des forces étrangères (alliées des mouvements rebelles) dans les processus de changement de régimes politiques en RD Congo, a conduit à beaucoup de dérapages de la part des chefs de guerre, donnant ainsi à penser que les projets de libération n'étaient autre chose que la consécration d'une nouvelle forme d'impérialisme au profit des groupes alliés étrangers (Amuri, 2012). Le RCD a mené une " guerre de libération » contre le régime de Laurent-Désiré Kabila, un pouvoir issu d'une première « guerre de libération » à la suite de la persistance des pratiques autoritaires sous le régime de Mobutu.

Le rôle joué par la troisième catégorie, les milices populaires maï-maï dans la plupart des provinces de l'Est en général, et la province du Maniema en particulier, est au centre de cette analyse. Il s'agit particulièrement de la propagande anti-tutsie menée à des fins d'endoctrinement et de recrutement de combattants, jeunes en majorité, dont les effets inattendus à l'égard de masses rurales, incultes en majorité, devraient attirer l'attention des pouvoirs 
publics, de la société civile, de l'opposition politique, des anciens miliciens devant être réinsérés dans la société, et de tous les autres acteurs (locaux, nationaux et internationaux).

Le nationalisme artificiel véhiculé par les enseignements idéologiques en direction des masses, représente une source potentielle de radicalisme violent et rampant que l'on peut redouter aujourd'hui à travers des demandes légitimes des jeunes (Brochard, 2016). Il faut y ajouter toutes les demandes faites au nom de la démocratie ou des droits de l'homme, dans un Etat devenu internationalisé à la suite des enjeux multiples et contradictoires que poursuivent divers acteurs. Au cours des deux dernières décennies d'instabilité multidimensionnelle, l'ampleur de la criminalité en milieu rural dans le Congo profond de l'Est est aisément associée avec la cristallisation de ce nationalisme de combat, identifiant tout étranger aux allures suspectes comme ennemi au même titre qu'un terroriste (Benhammou, 2017).

Cet article comprend deux principales parties: dans un premier temps, il précise le contexte et décrit le cadrage théorique fait des logiques et pratiques des acteurs qui se trouvent être les milices maï-maï, ainsi que la méthodologie proprement dite. Dans un second moment, il engage la discussion en analysant les trois documents retenus pour la mobilisation idéologique des masses en vue d'en saisir les glissements potentiels et réels vers la radicalisation, surtout chez les jeunes en quête de modèles de pensée et d'action dans un contexte de dénuement total.

\section{Considérations théoriques et méthodologiques}

De prime abord, il sied de rappeler que les africanistes ne cessent de s'interroger sur leur évaluation de la démocratisation lorsqu'ils sont frappés par le retour en force des autoritarismes dans bon nombre d'Etats africains. Collier et Levitsky (1997) sont ainsi surpris de déceler dans la littérature des exemples assez frappants d'invention sur le plan conceptuel visant à occulter le constat d'échec des processus de démocratisation. Ils notent qu'une première stratégie consiste à ajouter un adjectif pour qualifier la nature de la démocratie: "démocratie de façade », « démocratie autoritaire » et " démocratie militaire ». Ces chercheurs se plaignent que l'utilisation d'un adjectif dilue l'utilité analytique du terme de démocratie. D'autres estiment qu'il s'agit des régimes "hybrides" qui combinent des institutions démocratiques et des pratiques autoritaires (Diamond, 2002 ; Van de Walle, 2002).

Pour la longue période de transition ayant précédé ou suivi l'organisation des élections sur le continent, Céline Thiriot note avec pertinence : 
« En fait, si le début des années 1990 a été marqué en Afrique par une «vague » de démocratisation qui, comme une marée, a balayé nombre de régimes militaires, force est de constater que le ressac a été à sa mesure. Le Togo est un exemple de résistance et de restauration du pouvoir militaire. Les deux Congo (Brazzaville et Kinshasa) ont sombré dans la guerre, par choix délibéré des recours à la violence et de privatisation de force » (Thiriot, 2000, p. 202).

A la suite d'une étude sur les Etats en guerre en Afrique au cours des processus de démocratisation, Catharine Newbury résume la situation en ces termes :

« Bien que lourds de contradictions, les processus de libéralisation politique ont laissé espérer une forme de gouvernement plus responsable et plus sensible à la critique — et quelques pays africains ont obtenu des résultats impressionnants. Cependant, dans bien des parties du continent, les perspectives en ce début du vingt-et-unième siècle sont décidément plus sombres. L'augmentation de la violence et des guerres a eu des conséquences désastreuses pour les civils et les communautés auxquelles ils appartiennent » (Newbury, 2002, p. $1-2)$.

Pour sa part, Richard Banégas (2008) a étudié les milices en Côte d'Ivoire, en marge de ses enquêtes sur l'engagement des jeunes (Jeunes patriotes) dans la crise. Dans cette étude, l'auteur s'est interrogé sur la signification sociale et politique de ce phénomène milicien et de son devenir dans la situation post-conflit. A partir de l'observation des dynamiques de milicianisation qui traversent l'espace rural et celles de l'espace urbain dans le Sud du pays, il avance l'hypothèse que celles-ci participent à et de l'informalisation d'un Etat que l'on pourrait qualifier-sous certaines réserves-d' "Etat-milicien ». Rappelant le contexte socio-politique de production des milices, Banégas précise :

« Depuis le début du conflit, en septembre 2002, la Côte d’Ivoire s'est engagée dans un processus multiforme de «milicianisation » de la société, de privatisation de la violence et de militarisation de la jeunesse sous la houlette du régime FPI. (...) Dès le début des offensives rebelles dans l'Ouest à l'automne 2002, le Président Gbagbo et ses conseillers ont pris conscience qu'ils ne pourraient guère compter sur l'armée nationale, affaiblie et divisée, pour résister et éventuellement reconquérir le territoire » (Banégas, 2008, p. 1).

La problématique de la démocratisation en Afrique permet de comprendre le sens des interventions militaires et des milices dans le processus de changement politique en RDC pendant la transition. Le régime de Laurent-Désiré Kabila, d'essence militaire, a été accueilli avec enthousiasme au détriment du long processus démocratique qui était en cours 
au pays sous Mobutu grâce aux partis d’opposition et à la société civile en formation. Les mêmes causes produisant les mêmes effets, les carences démocratiques très tôt remarquées dans le chef du Président Kabila, qui justifiait la guerre par des impératifs de démocratisation, ont suscité une seconde guerre sous le même label de libération de la démocratie.

C'est dans ce contexte de privatisation de la violence ou sa démocratisation (Amuri, 2007), que les milices maï-maï apparaissent dans le Maniema rural pour affirmer politiquement le refus des populations face au régime rebelle du RCD et ses alliés rwandais (Amuri, 2004).

Parmi les stratégies de mobilisation utilisées par les Maï-Maï, nous avons retenu celle de l'exploitation de l'écrit en tant qu'instrument de propagande et de contre-propagande. Essentiellement, il s’agit de saisir la signification et les effets des messages articulés autour du rôle suspect (« impérialiste ») qui serait joué par ce mouvement politico-militaire soutenu par le Rwanda (Amuri, 2008a). Cette stratégie, tout en propulsant le mouvement milicien à un niveau de légitimité jamais imaginée au départ, a débouché sur une politisation excessive de masses rurales. La reconfiguration du champ social à la suite de la propagande entreprise par la manipulation des documents dénonçant les faits d'impérialisme dont le RCD serait le vecteur, semble remettre en question ou, du moins, élargit la thèse de Théodore Holo qui lie, de façon étroite, le phénomène de pouvoir [politique] au milieu urbain :

«Il n'est un secret pour personne, en tout cas pas pour les politologues, que le pouvoir en Afrique est un phénomène essentiellement urbain. Le pouvoir est exercé par les élites occidentalisées, à telle enseigne qu'on observe un divorce entre les détenteurs du pouvoir politique et la société. Il y a cohabitation de deux légitimités : l'une moderne et urbaine, l'autre traditionnelle et rurale » (Holo, 1994, p. 121).

Comme on peut le constater, le travail à effectuer au niveau des populations rurales constituées en majorité de paysans apparaît ici crucial en vue de la mobilisation effective de ces derniers. Tout en contestant la thèse de Frantz Fanon selon laquelle « dans les pays africains, seuls les paysans sont révolutionnaires », Romano Ledda conclut que la situation des campagnes requiert un travail de mobilisation et d'organisation complexe que «seule une force extérieure à la campagne » (Ledda, 1967) pourrait accomplir. Les paysans ne peuvent donc pas jouer le rôle de pointe dans la lutte politique. Cependant, comme le note Faustin Mulambu, «nous ne possédons que très peu de travaux sur la participation paysanne dans les mouvements sociaux en Afrique noire » (Mulambu, 1970, p. 439).

D’un point de vue strictement méthodologique, il est essentiel de signaler que la critique historique reposant sur l'heuristique (rassemblement des matériaux d'analyse : essentiellement les documents écrits ou textes) et 
l'herméneutique (interprétation des documents sur le plan externe et sur le plan interne) a été mobilisée aux fins de l'examen des documents destinés à la mobilisation idéologique de masses rurales au Maniema. En effet, la critique extrinsèque (externe) des textes implique la prise en considération de leur authenticité, de leur intégrité, de leur date et de leur origine ou provenance. Notre attention est focalisée sur les caractères diplomatiques ${ }^{12}$ et philologiques ${ }^{13}$. La critique intrinsèque (interne) concerne la vérification des réponses à deux questions essentielles : l'auteur ne s'est-il pas trompé ? N'a-t-il pas voulu tromper les lecteurs ? En rapport avec ces deux dernières dimensions (questions), il est fondamental de noter que les documents examinés reflètent un radicalisme qui est de nature à susciter le doute de lecteurs critiques; en revanche, ces documents exercent un effet de persuasion remarquable à l'endroit des masses, déjà conditionnées psychologiquement, dont les regards sont tournés vers les conséquences de la guerre sur le terrain, leur milieu de vie. L'on constate, pour l'essentiel, que les documents sont taillés sur le modèle de tracts avec une rhétorique orientée vers l'identification de l'ennemi commun, le bourreau, l'envahisseur...

Ces documents, écrits en français, sous examen sont les suivants :

- $\quad$ Le rêve fou de Kagame : le Congo dépecé ;

- $\quad$ La réunion tenue en Amérique. Document Top secret. Processus de la guerre de conquête des Tutsi «Bahima »;

La Charte de l'impérialisme.

En termes de sources, les combattants maï-maï soutiennent que les documents qu'ils exploitent ont été trouvés chez leurs ennemis : les uns chez des sujets rwandais tombés sur le champ de bataille, les autres chez des sujets congolais qui n'ont pas su les garder ou, du moins, leur accorder toute la discrétion voulue en raison de la conjoncture sociopolitique de l'époque.

Il est également important de noter d'un point de vue ethnographique que les populations de la province du Maniema, dans leur majorité, ont la réputation d'être violentes (Amuri, 2004). A juste titre, ceci est intéressant pour qui veut comprendre comment la propagande contre les rebelles et leurs alliés rwandais, ne pouvait que réactiver le réflexe contestataire de ces communautés locales. Voici comment un témoin de l'ancienne rébellion de vengeurs de Lumumba au Maniema rapporte des précédents historiques sur la tradition guerrière de certains groupes et l'héritage inachevé de la pacification entreprise dans cette vaste jungle:

\footnotetext{
${ }^{12}$ Les formules initiales et finales, les titres donnés aux personnages, les institutions, les coutumes ou usages signalés dans les textes.

${ }^{13}$ Particulièrement, la langue et le style utilisés pour déceler les interpolations.
} 
« Autour de l’Urega, pays des Warega, se pressent de nombreuses tribus turbulentes. En particulier les Bakusu, Wasimba, Bango-bango et Babembe, où les arabisés ont fait souche et proliféré ! Mais encore les Bakumu, Wasongola, Bashi et Bakwame ! Cet assemblage hétéroclite parle des dialectes différents. Les clans sont étroitement cloisonnés, les intérêts divergent. Le colonisateur a difficilement pacifié et maintenu l’ordre dans la région. Maintenant, tout cela fermente. Ces gens n’attendent que le moment propice pour se rentrer dedans ! » (Adam, 1999, p. 27).

En termes de présentation du milieu d’étude, il est pertinent de noter que le terme Maniema vient du terme «Nyuema» qui, en dialecte bangubangu des populations vivant dans la contrée de Kabambare, signifie «forêt » ou mieux «pori » en swahili local. Cette thèse rejoint celle de Hosten qui, en s’appuyant sur les écrits de Livingstone, concluait en 1957 dans son travail que le terme «Maniema » signifiait " pays de la forêt ». Ceci résiste au doute malgré la position de l’historien contemporain Isidore Ndaywel, citant Haddad, qui pense le contraire en disant: «Le mot Maniema dont on pensait qu'il voulait dire le 'pays des forêts' rempli des 'mangeurs d'hommes' serait simplement d'origine arabe (Manâma) et signifierait 'lieu où l'on dort', 'pays où l'on vient se reposer' après des razzias opérées dans la périphérie ». Donc, l'appellation de Maniema est antérieure à l'arrivée des Arabo-Swahili puisque les populations autochtones l'utilisaient pour désigner un milieu naturel de leur contrée à savoir, la forêt (N'Sanda, 2004).

On ne peut, dans ces conditions, s’étonner de constater que la ruralité constitue encore une caractéristique dominante de vastes territoires moins peuplés du Maniema. Cette province est comprise entre 0 et 5 de latitude Sud et 24 et 28 de longitude Est soit une distance à vol d'oiseau de $525 \mathrm{~km} \mathrm{du}$ Nord au Sud et $450 \mathrm{~km}$ de l'Est à l'Ouest. Avec une superficie de 132 776km2, soit 5,6\% de superficie totale du Congo. Le Maniema est limité au Nord et au Nord-Est par la province de la Tshopo, au Sud par la province du Tanganyika et la province de Lomami, à l'Ouest et au Sud-Ouest par la province du Sankuru, et à l'Est par les provinces du Nord et Sud-Kivu

Dans cette étude, parler de «masses rurales » ne consiste qu’à souligner le caractère ou l'ampleur du statut subordonné des masses vivant en milieu rural. Puisque le rural est généralement assimilé au paysan et naturellement subordonné à l'urbain, il va de soi que les élites rurales (par rapport au concept 'masses rurales') demeurent, elles aussi, subordonnées à celles urbaines comme la périphérie l'est au centre en matière de pouvoir. Le concept de masse, que l'on oppose généralement à celui d'élite, est utilisé simplement au sens de groupe de populations, de communauté, bref, d'un ensemble de personnes marquées par la conscience d’être dominées ou, du 
moins, subordonnées par rapport à une instance de pouvoir politique. Cette masse, censée être majoritaire, est caractérisée du point de vue idéologique par une homogénéité certaine et prégnante quant aux représentations et perceptions que se font ses membres du pouvoir détenu par la minorité au sein de la société.

En privilégiant l’analyse du phénomène Maï-Maï en tant que réalité de violence politique, les concepts 'rural' et 'paysan' sont ici utilisés indifféremment en raison de l'importance accordée à la problématique de la démocratisation en tant que processus d'intégration devant prendre en compte les espaces isolés et les intérêts divers que représentent les milieux ruraux $^{14}$. En fait, ces milieux sont habités majoritairement par des paysans, ceux-ci étant généralement identifiés comme des populations pauvres, analphabètes et dépolitisées dans la plupart des Etats africains. Cet enjeu du pouvoir, ou ce besoin d'expansion de l'espace démocratique, est bien manifeste à travers le titre de l'ouvrage de Koffi : Le pouvoir de la brousse. Ni démocratie ni développement en Afrique noire sans les paysans organisés (Koffi, 1993).

\section{Comprendre le statut des acteurs et les effets de l'écrit en politique pendant la guerre}

La section comporte quatre points essentiels. Le premier point, relativement long, fait une description offrant une vue panoramique sur les acteurs maï-maï en tant que milices marquées par une origine idéologique, géographique, ethnique... diverse, même s’ils partagent tous une origine sociale paysanne, ce qui contribue à l'homogénéisation (apparente) de leur identité associée voire assimilée à celle des masses rurales ${ }^{15}$. Il s’agit donc d'un aperçu sur l'identification du mouvement maï-maï dans l'espace géopolitique du Maniema contemporain. Les trois autres points se focalisent sur les matériaux formant le corpus exploité par le mouvement maï-maï pour la cause idéologique. Ils correspondent aux trois documents retenus pour le décryptage des processus de dénonciation des pratiques des adversaires et de la politisation de masses rurales pendant les années de guerre au Maniema ${ }^{16}$.

\footnotetext{
${ }^{14}$ Allusion est faite à la notion d'expansion ou élargissement de l'espace démocratique.

${ }^{15}$ Nos recherches antérieures avaient déjà abordé cette dimension de l’origine sociale des miliciens maï-maï. Les miliciens mä̈-maï sont d'une origine sociale essentiellement paysanne et accusant un déficit profond en matière d'éducation moderne (l'école occidentale) pour la grande majorité de cas.

${ }^{16}$ Ces documents ont déjà été annoncés sous la rubrique "Considérations théoriques et méthodologiques"'.
} 
Saisir l'origine idéologique, magico-religieuse, géographique, ethnique... des milices maï-maï à travers l'historicité des héritages

En se référant aux recherches historiques, dont celles de N'Sanda (2003b) en particulier, l'on ne peut s'empêcher de reconnaître que le terme Maï-Maï a déjà été utilisé à plusieurs reprises dans la sous-région des Grands Lacs. A l'époque coloniale, il a servi à désigner la révolte d'un mouvement magico-religieux qui, en Afrique orientale (qui deviendra plus tard après la guerre de 1914-1918, le Tanganyika Territory), eut des démêlées avec l'administration coloniale allemande entre 1905 et 1907 à savoir, le mouvement « Maji-Maji » dirigé par le " prophète » Kinjikitele. A la suite de la répression dont il fut l'objet, ce mouvement disparut près d'un demi-siècle.

Ce nom réapparaît au Congo-Kinshasa ${ }^{17}$ en 1964 avec la rébellion muleliste que décrit si bien Benoît Verhaegen dans son livre « Rébellions au Congo ». Les guerriers de cette rébellion s'appelaient "simba" (terme swahili désignant le lion, animal choisi comme totem) et leur cri de guerre était "mulele maï ». Selon leurs croyances, les guerriers «simba » ne devaient subir aucun effet de balles tirées par leurs ennemis qui se transformaient plutôt en gouttelettes d'eau. Cette même croyance était déjà présente dans la révolte des Maji-Maji de Kinjikitele dans le Tanganyika allemand.

Il faut reconnaître d'ailleurs que la pratique magico-religieuse de la rébellion muleliste n'est pas un fait nouveau dans les révoltes congolaises. A l'époque coloniale, d'autres mouvements y ont eu recours, notamment « la révolte pende » dans le Bandundu en 1931 et la "révolte de Kitawala » menée par Bushiri en 1944 dans les territoires de Masisi, Lubutu et Punia.

Après l’indépendance en 1960, le cri de «maï » apparaît pour la première fois avec les milices nationalistes radicales d'obédience lumumbiste qui opèrent à l'Est du Congo et évoluant sous le label de " Cartel ». Le plus connu de ce mouvement est le «Cartel Balubakat » qui fut présent au Nord-Katanga et au Maniema entre 1960 et 1963 . Le rituel d'immunisation des « jeunesses » de ce mouvement s'appelait " kuoka maï » ( «se laver à l'eau»). Comme le rapporte Benoît Verhaegen, le rituel consistait en l'aspersion du candidat-jeunesse d'eau magique et en des scarifications enduites de «dawa ». "Kuoka maï» peut être traduit en termes de passage à travers "un baptême par l'eau magique ", de l'état vulnérable du simple «civil» (paysan ou autre) à celui du guerrier immunisé, fort et invincible. Plus de quarante ans après, ce nom refait surface avec le mouvement Mä̈-Maï » à la suite de la guerre qui ravageait le Kivu montagneux et le Maniema.

\footnotetext{
${ }^{17}$ Entendez, la République Démocratique du Congo.
} 
Il est intéressant d'observer la constance de l'association entre les mouvements pratiquant ce rituel et les espaces ethniques. Benoît Verhaegen le souligne d'ailleurs dans le cas des «simba» :

«La puissance des dawa, écrit-il, dépendait principalement de leur origine: parmi les populations favorables aux simba, quatre groupes ethniques avaient la réputation de posséder des traditions médicales supérieures aux autres: les Baluba du Nord-Katanga, les Bembe, les Wazimba et les Bakusu-Batetela » (Cité par N'Sanda, 2003b).

En effet en 1960, les « jeunesses » du « Cartel » combattant au NordKatanga et au Maniema sont en majorité issues de l'ethnie luba du Katanga. Et les pratiques rituelles qu'ils utilisent sont tirées de leur patrimoine traditionnel dit "Bwanga bwa mpundu ». Plus tard en 1964, les «Simba » qui partent à l'assaut du Maniema sous le commandement d'Olenga (un Tetela du Sankuru), sont les Babembe de Fizi et leur « dawa » provient de leur terroir. Les Balega de Mwenga qui combattent les rebelles mulelistes à Kitutu en territoire de Mwenga au Kivu en 1964, utilisent un «dawa » tiré de leur territoire. Et lorsque les populations du Maniema sont entrées dans cette rébellion muleliste, les « dawa » qu'ils allaient utiliser provenaient des Bakusu-Batetela et des Binja. La féticheuse en chef de toute la rébellionMama Onema—était d'ailleurs une «Tetela » de Sankuru.

Pendant la guerre de 1998, les mêmes « dawa » qu'utilisent plusieurs groupes en lutte contre la rébellion du Rassemblement Congolais pour la Démocratie (RCD) et l'armée rwandaise d'occupation dans l'Est du pays, proviennent avant tout des terroirs des combattants. Les Maï-Maï bembe opérant dans le Fizi et Uvira (Sud-Kivu), tout comme les Binja opérant au Maniema et les Nyanga-Hunde de Masisi-Walikale (Nord-Kivu), utilisent chacun, un «dawa » de leurs ethnies respectives (N'Sanda, 2003b).

A l'instar des autres provinces de la RDC où il s'est manifesté, le phénomène Maï-Maï au Maniema trouve son origine dans le contexte de la guerre de 1998, politiquement perçue comme inopportune. Cette guerre à forte pondération externe car caractérisée par la prépondérance des alliés étrangers et l'extraversion de son leadership ${ }^{18}$, a poussé les populations rurales à l'activisme. Celui-ci, exclusivement mené par des autochtones, comporte des caractéristiques propres reflétant celles des leaders et des espaces géographiques dans lesquels il se déploie. En d'autres termes, le phénomène Maï-Maï au Maniema comprend trois trajectoires correspondant aux trois entités décrites en termes de pools à savoir le Maniema

\footnotetext{
${ }^{18}$ Il était courant d'entendre les observateurs déplorer le fait que ce sont les étrangers qui avaient la préséance sur leurs alliés congolais dans tous les domaines relevant du Rassemblement Congolais pour la Démocratie (RCD).
} 
septentrional ${ }^{19}$, le Maniema central $^{20}$ et le Maniema méridional ${ }^{21}$. Cependant, pour une caractérisation systématique, il y a lieu de considérer une structuration basée à la fois sur l’ordre chronologique d'apparition et la proximité idéologique des milices maï-maï en termes de générations. Pour rappel, les enquêtes de terrain conduites au Maniema dans le passé (Amuri, 2004, 2007, 2008a), permettent de distinguer les types ci-après :

- $\quad$ Premièrement, les milices dont les sites et le leadership rappellent les rébellions antérieures marquées par le nationalisme radical des partisans lumumbistes et mulelistes ;

- $\quad$ Deuxièmement, les milices dont les sites, le leadership et l’idéologie renvoient principalement à la perspective messianique ;

- Troisièmement, enfin, les milices dont les sites, le leadership et l'idéologie correspondent à l'effet de diffusion et de dégénérescence du phénomène milicien, conduisant ainsi à sa recomposition.

Le premier type de milices rappelle les nationalistes de la première heure pour la période postcoloniale. Les termes "lumumbistes" et "mulelistes" signifient respectivement les partisans de Patrice-Emery Lumumba, premier Premier Ministre du Congo indépendant, et de Pierre Mulele, ancien ministre de l'Education nationale dans le gouvernement Lumumba en 1960. Les rebelles de cette première vague, étaient tous identifiés comme des «vengeurs de Lumumba » assassiné en 1961. Les milices constituées autour d'anciens rebelles se réclamaient ainsi de l’idéologie nationaliste de Lumumba, localisées dans le Maniema méridional sous le commandement de colonel Raphael Milambo Komombo, sujet zimba (Binja-Sud), dans le territoire de Kasongo, et le Maniema septentrional sous le commandement de colonel Grégoire Kayobo, sujet kumu, dans le territoire de Lubutu.

${ }^{19}$ Le Maniema septentrional comprend les ethnies traditionnellement alliées que sont les Balega du territoire de Pangi, les Wasongola (Binja-Nord) du territoire de Kailo, les Bakumu, les Lengola-Mituku des territoires de Punia et Lubutu. Cette partie est essentiellement dominée par le christianisme

${ }^{20}$ Ce pool comprend principalement le territoire de Kibombo et la ville de Kindu. Le territoire de Kibombo est essentiellement la contrée des Bakusu et des leurs "cousins » Bangengele. Cinq entités administratives dites «collectivités locales » regroupent chacune une communauté clanique: les Bahina, les Matapa, les Bakongola, les Ankutshu et les Bangengele. Le Maniema central est le lieu de la symbiose de toutes les valeurs, allant des aspects ethniques à ceux politiques, religieux ou socioculturels et économiques.

${ }^{21}$ Ce dernier pool comprend les territoires de Kasongo et de Kabambare. Contrairement aux territoires précédents qui, hormis la ville de Kindu, sont presque mono-ethniques, le Maniema méridional possède une grande diversité ethnique. Le territoire de Kasongo comprend huit ethnies regroupées au sein des entités administratives dites «collectivités locales ». Il s’agit de : Nonda, Wazura, Zimba (Binja-Sud), Bakwange, Wagenia, Basongye, Babangubangu et Batabwa. Cette partie du Maniema est marquée par l’Islam comme religion. 
Le second type de milices correspond aux milices idéologiquement inspirées de chefs de guerre prétendant jouir du statut de "prophètelibérateur"' du fait de leur capacité à réinventer le messianisme connu pendant la colonisation. C'est le cas des miliciens dirigés par Kabambe wa Kabambe, sujet genia, dans les territoires de Punia et de Kailo, particulièrement avant la réunification des milices du Maniema. Concernant ces milices ayant connu des chefs de guerre à statut de "prophètelibérateur“, il convient de souligner l'impact non négligeable que les mouvements messianiques ont exercé sur les territoires situés au Nord du Maniema pendant la période coloniale. Il s'agit notamment du Kimbanguisme (du nom du prophète noir Simon Kimbangu) dans la contrée riveraine de Lowa le long du fleuve Congo et du Kitawala (déformation du Watch Tower américain ayant subi des influences locales) dans les territoires de Lubutu et Punia particulièrement.

En effet, la plupart des relégués de ces deux mouvements ont été identifiés comme des acteurs-clés du radicalisme rural dans les milieux d'implantation des entreprises d'exploitation minière et des plantations des colons blancs (Lovens, 1974; Mwene-Batende, 1982). Même après l'indépendance, les mouvements incarnant la contestation sociopolitique du néocolonialisme, notamment les rébellions nationalistes ont été vues comme découlant du même moule idéologique que lesdits mouvements messianiques d'inspiration chrétienne. La convergence de tous ces facteurs (politiques, ethniques, religieux, socioculturels, économiques,...) a ainsi tendance à renforcer l'opinion populaire sur le caractère structurel et atypique de la violence au Maniema (Amuri, 2008a). La continuité historique entre les mouvements messianiques de la première heure (période coloniale) et le mouvement Maï-Maï du Maniema est attestée par des faits. Elle ne fait donc pas l'objet de doute dans la mesure où l'analyse des corpus doctrinaux et des pratiques révèle, non seulement l'identité des rapports de domination en termes de dialectique impérialistes-nationalistes ou bourreaux-victimes, mais aussi la constance des attitudes et comportements dans les espaces de violence en effervescence correspondant aux origines des acteurs dont principalement les leaders : origine ethnique, sociale, religieuse ou culturelle.

Le troisième type de milices est simplement identifé avec toutes les milices nées sous l'effet de l'expansion du mouvement maï-maï à partir du centre (la ville de Kindu, chef-lieu de la province du Maniema) pour s’installer dans les territoires de Kasongo, Pangi et Kibombo (sites dominés par la milice «Kaka Sawa » de Kalume dit Kiwis). L'on assiste aussi à la multiplication des groupes miliciens restreints et homogènes ethniquement dans chaque contrée, du Nord au Sud du Maniema. Ces milices incarnent les agendas cachés de certains groupes ethniques ou tribus minoritaires qui se sentaient, jusque-là, marginalisés des structures politico-administratives de la 
province. C'est particulièrement le cas des Balanga, Bangengele, Bahombo, Basongola qui ne sont pas représentés d'une manière significative dans les instances provinciales de décision (Amuri, 2004). La plupart de ces groupes sont localisés dans les territoires qui ont fourni des effectifs importants de miliciens ${ }^{22}$.

Cette génération de miliciens correspond à la pré-transition du mouvement maï-maï qui, rapidement, sera marquée par des changements majeurs : unification des milices en un seul mouvement, négociations entre le Gouvernement de Kinshasa, les mouvements et factions rebelles, les milices maï-maï, les milices ethno-politiques hema et lendu de l'Ituri (dans l'ex-Province Orientale) et la société civile, sous l'égide de la Mission de l'Organisation des Nations Unies en RDC dans sa phase d'observation. Ce processus politique ayant emmené les négociateurs de ces différentes composantes et entités au Dialogue inter-Congolais en Afrique du Sud (Sun City), conduira à la signature de l'Accord Global et Inclusif et à la mise en place des institutions politiques de la transition à laquelle certains chefs maïmaï participeront avec statut d'hommes politiques tandis que la plupart d'anciens miliciens (combattants) rejoindront l'armée nationale lors des opérations de brassage des forces. Plus tard, les ex-miliciens devenus hommes politiques, se présenteront successivement aux élections de 2006 et 2011 même si leur sort n’aura pas été brillant ${ }^{23}$.

Les réflexions qui précèdent permettent de confirmer que le besoin de politisation de masses rurales, donc de participer à la vie politique, surtout après avoir enregistré beaucoup de frustrations du fait des actions traduisant une domination illégitime de la part de ceux qui occupent le centre (le pouvoir politico-administratif dans les villes), contrôlent le gros de ressources et disposent du pouvoir de vie et de mort à l'égard des autres groupes, est à considérer comme un phénomène universel.

En effet, les populations du Maniema ont connu des expériences tragiques pendant la guerre du RCD avec ses alliés rwandais. Les conditions psychologiques (traumatismes de guerre, étranger chez soi, humiliation...) et matérielles (misère généralisée, absence de services sociaux, carence de produits de première nécessité...) étaient réunies pour que ces populations

${ }^{22}$ Parmi les noms les plus cités par les personnes interrogées à Kindu lors de nos enquêtes de 2006, il y a Lambert Konga Kanape, Satana, Dunia Dalos, Lukudja, Lumanisha (Lumaniza) dit PDG, Charles Mozon. Certains noms ne sont en fait que des sobriquets adoptés par les intéressés pour leur sécurité. Ces opérateurs de la violence adoraient aussi l'astuce consistant à ne livrer qu'un seul nom, laissant ainsi le doute s'installer dans les esprits des agents de renseignement du RCD qui se mettraient sur leur trace.

${ }^{23}$ Faute d'une préparation sérieuse en matière de leadership et de compétition politique, ce qui est devenu plus difficile dans une démocratie qui empêche tout renouvellement effectif de la classe dirigeante, l'échec politique des élites d'origine milicienne et rurale était simplement sans surprise. 
non seulement se montrent sensibles à la propagande menée par les miliciens maï-maï, mais aussi et surtout s'intéressent à la politique et s'y engagent résolument afin de tester leur citoyenneté.

Parmi les acteurs impliqués de manière active dans les milices maïmaï, que l'on pensait dominées par les groupes ethniques à tradition guerrière, l'on a pu repérer certains éléments, bien que formant une minorité, qui relèvent de groupes ethniques réputés pacifiques. Preuve que très souvent les groupes n'attendent que des circonstances déterminantes (bonnes ou mauvaises) et surtout une impulsion (l'initiative d'un leader charismatique), pour s'activer. C'est ce qui ressort de ce témoignage historique sur la propagande des nationalistes pendant la première rébellion dans le Maniema:

«Les missionnaires avaient sous-estimé les effets de la propagande politique nationaliste en milieu rural et surtout parmi les Warega, qu'ils croyaient dociles et acquis à toute formation politique modérée s'opposant à l'hégémonie des politiciens de Kindu [ici les nationalistes et radicaux, Bakusu et Batetela] » (Verhaegen, 1969, p. 99).

L'ampleur des effets qu'un enseignement idéologique peut avoir sur les masses dans un contexte spécifique comme celui de guerre, est bien perceptible dans les contenus livrés par les documents analysés dans les trois points qui suivent.

\section{"Le rêve fou de Kagame : le Congo dépecé"}

Labélisé 'L'œil du peuple' comme identité de l'auteur, ce document d'une page est présenté comme ayant été produit à Bunyakiri (au Sud-Kivu) en date du 25 Juin 1999. Dès le début, il est dramatiquement souligné que le danger imminent qui guette le pays et le peuple congolais, ne permet aucun doute quant à la nécessité et l'urgence d'une action collective de sauvetage de la part des Congolais en général et de la population du Kivu en particulier. En fait, comme cela transparaît nettement dans l'extrait qui suit, c'est le discours de l'autochtonie qui fonde la mobilisation des masses urbaines et rurales en vue de sauver les terres ancestrales, le terroir menacé qu'est le Kivu :

"Congolais, population du Kivu! Le danger est là, il guette, il menace, et se prépare à dévorer toute la nation congolaise. La carte [cidessous] Que nos services ont interceptée circule encore sous les manteaux à Kigali et à Goma. Elle est la cause d'éclatement du RCD. Elle illustre le sort qu'attend notre pays et notre peuple (sic) ».

Le document comporte une carte avec une légende indiquant le découpage qui en est proposé et le calendrier d'exécution du plan de démembrement effectif du Congo en zones d'influences par les armées rwandaises, ougandaises et burundaises suivant cinq étapes. Ainsi, peut-on y lire : «Ce plan Kagame surnommé dans certains milieux rwandais ‘opération 
Berlin II’ et soutenu par quelques traîtres congolais, va se réaliser en cinq étapes ».

Pour une meilleure lecture de la propagande basée sur le crédo de la toute-puissance de l'écrit, nous reprenons en intégralité le chronogramme proposé en vue de la réalisation du plan dit Kagame.

En voici les étapes :

1. «Le 30 juin 1999: La proclamation de la province autonome ou fédérée du Kivu, par les responsables du RCD aile Ilunga, Kagame interposé. Ainsi le Rwanda circuite les accords de paix de Lusaka (sic).

2. 1 janvier 2000 :

- $\quad$ Mise en place de l'assemblée provinciale autonome qui aura pour mission principale de légitimer la sécession de Kivu ;

- Début officiel de l'implantation massive et inondation démographique dans le Sud-Kivu par les tutsi (cfr Muhumba, Nguba, Nyawera etc...). Le démantèlement des entités non tutsi et séparation physique entre les parties tutsies et autochtone plus particulièrement à Bukavu, Uvira, Goma, et dans le Haut-Plateau d'Itombwe (sic) ;

- $\quad$ Chasse aux non-originaires, les plus visés sont: Les Katangais, Kasaïens, Equatoriens. (La marionnette Kantitima l’a déjà inauguré par la chasse des fonctionnaires non-originaires dans les maisons de l'Etat à Bukavu) ;

- $\quad$ Début de construction de 600.000 maisons qui seront financées par la Banque d'Implantation Tutsi sous forme de crédit, de commerce et d’administration des colons. (cfr Les accords de Lemera que Kabila a refusé de mettre en application).

3. 1 janvier 2005 :

- Avec les Tutsi majoritaire, organisation d'un référendum d’autodétermination de peuple du Kivu qui sera suivi par la proclamation de l'Etat libre du Kivu (sic) ;

- Retrait des troupes rwandaises dans le Katanga et le Kasaï en échange de la reconnaissance du nouvel Etat libre (échange de territoire contre la paix) avec ce qui va rester du pouvoir de Kinshasa ;

- $\quad$ Mise sur pied du projet d'exploitation économique du Kivu : début de l'exploitation des riches gisements de pétrole qui s'entend ${ }^{24}$ entre les régions Béni au Nord-Kivu jusqu'à la partie congolaise de Gatumba au Burundi (sic) ;

- Construction d'un pipeline entre Gatumba et Dar-es-Salam et Lubero-Mombasa.

\footnotetext{
${ }^{24}$ Le contexte indique qu'il s’agit bien de “s’étend” (verbe: s’étendre, et non entendre).
} 
4. $\quad 2$ septembre 2007 : Proclamation de la confédération des Etats du Kivu, du Rwanda et du Burundi, qui sera appelé la Confédération des pays des Grands Lacs (sic).

5. 1 janvier 2010 : Fin de la confédération bidon, retrait du Burundi, Kivu $10^{\mathrm{e}}$ préfecture du Rwanda ».

A la fin du chronogramme présenté ci-dessus, une mention spéciale est faite en termes d'interpellation en direction des Congolais et Congolaises c'est-à-dire les fils et filles du pays. Clairement, c'est l'action salutaire de ces derniers est attendue sans délai s’ils prétendent encore avoir quelque attachement sincère à leur patrie qui était plus que jamais en danger face aux « expansionnistes rwandais » (ici les Tutsi). Il est précisément noté ceci :

«Ainsi, chers compatriotes, la boucle sera bouclée et la colonisation tutsi sur notre pays sera effective.

Que va être notre sort, le sort de notre progéniture et de notre pays?

Allons-nous continuer à croiser les bras face à l'arrogance des Tutsi et de leurs valets?

Allons-nous continuer à supporter les diatribes qu'un piètre Avocat Mudimbi déverse en longueur de journée à la radio et à la TV ?

Allons-nous laisser l’intégrité de notre territoire sous le contrôle des agresseurs Tutsi ?

Suivons l'exemple de nos jeunes enfant qui face à l'Armada militaire de Tutsi n'hésitent pas à s'opposer aux manœuvres de RCD tendant à nous imposer le drapeau de Kagame » (sic)

L'observation pertinente à faire sur les extraits ci-dessus, c'est que le discours sur un éventuel processus Berlin II visant le redécoupage des certaines régions d'Afrique comme pour la Région des Grands Lacs, ne procède pas d'une simple utopie. Il reflète en partie la réalité de certains Etats, le Rwanda en l'occurrence qui est principalement impliqué dans la logique de conquête en vertu du principe de l'espace vital. Avec sa capacité militaire, il a réussi à maintenir sa présence sur le territoire congolais depuis la guerre de 1996-1997 et à en extraire des ressources diverses.

Remontant le passé pour comprendre la ferme conviction des populations de l'Est et leur refus radical face au projet d'occupation du Kivu par l'alliance tutsie, Emmanuel Lubala Mugisho note :

«Depuis la première guerre du Kivu en 1996, les populations de cette région avaient suspecté l'engagement des troupes rwandaises, ougandaises et burundaises aux côtés des rebelles banyamulenge, notamment à cause des déclarations du Président Bizimungu du Rwanda révélant les ambitions territoriales de son pays sur le Kivu. En effet dans son discours du 10 Octobre 1996 à Cyangugu celui-ci affirmait "Même dans la région appelée Bishugi considérée aujourd'hui comme le berceau Banyamulenge, tous les habitants de 
ces contrées étaient des Rwandais. Même Kayenzi et les autres, situés actuellement au Zaïre faisaient partie du Rwanda » Ainsi, pendant que la presse internationale s’empressait à faire accréditer la thèse d’une « guerre de libération », les populations du Kivu, autres que les Banyamulenge, avaient la conviction de subir « une guerre d'occupation ».Et les faits ne les ont pas contredits.» (Lubala, 2000, p. 200-201).

Plus loin, soulignant la volonté d’occupation comme une donnée permanente de l'invasion du Kivu, l'auteur rappelle longuement ceci :

«Le Kivu représente un enjeu important pour le Rwanda, le Burundi et l'Ouganda pour des raisons stratégiques, sécuritaires et économiques. Dans la lutte pour le leadership régional que se livrent le Rwanda et l'Ouganda, tenir sous sa coupe une partie ou la totalité du riche territoire congolais est le moyen le plus sûr de devenir une puissance. C'est pourquoi d'aucuns estiment que, pour conforter son ambition, le général Paul Kagame « a besoin de stabiliser son environnement immédiat » dont ferait partie le Kivu (...) si le contrôle du Kivu leur ( Rwanda, Burundi et Ouganda) permettrait de réduire la menace de déstabilisation de leurs rébellions respectives qui s’y trouvent, il leur donnerait également l'occasion de disposer du diamant et de l'or, mais aussi des métaux précieux employés dans la technologie informatique.» (Lubala, 2000, p. 201202).

Recentrant le débat au Maniema en tant que champ d'observation spécifique du phénomène milicien dans notre étude, il y a lieu de noter avec Raymond Emmanuel Mutuza Kabe, citant Lumuna Sando, un précédent non négligeable sur la crainte d’occupation tutsie sous la deuxième République. Il écrit ceci :

« Il s’agit aux yeux des Zaïrois (...) d'une guerre de conquête territoriale d'une forme particulièrement insidieuse ! En effet, sans chercher immédiatement une quelconque annexion du territoire, la conquête tend à organiser d'abord l'implantation des colonies rwandaises sur des territoires zaïrois. (...). Sous cette forme, reprécise Lumuna, la guerre de l'Est comporte plusieurs fronts qui révoltent et qui laissent tout Zaïrois dans une frustration profonde : un front militaire - c'est nous qui soulignons - un front militaire où le Zaïre, malade de sa transition et de son armée doit aussi faire face à des puissantes forces internationales; un front médiatique, où l'on enferme déjà le Zaïre dans le piège du génocide, d'atteinte aux droits de l'homme, etc. , un front culturel où l'on accule les Zaïrois soit apparaître comme des vilains xénophobes, soit à devoir dénoncer une soi-disant tribu qui n’a jamais été des leurs ni avant la 
colonisation, ni dans l’ Etat indépendant du Congo, ni dans le Congo Belge ; en récusant tout désir de Prince, il doit bien soumettre les réfugiés et les immigrés présents sur son territoire au même traitement que dans tous les pays du monde, et cela quelle que soit la durée de cette présence ; un front idéologique, enfin où l’on pousse en avant le prétexte de libération ou de changement de régime, voulu par les agresseurs,... un changement qui, malgré la longue transition menée par les seuls Zaïrois et le calendrier électoral, prévu pour 1997, devrait ainsi subitement apparaître comme un cadeau de Rwandais... un cadeau qui aurait pour prix la reconnaissance de la nationalité zaïroise aux immigrés Tutsi collectivement »(Mutuza, 2004, p. 8990).

Qu'on en soit convaincu ou pas, les nombreuses sources documentaires rassemblées pour étayer notre argumentation nous placent devant un embarras réel : certaines sources ont une valeur de pure propagande et semblent alimenter la crise plutôt que de l'atténuer; d'autres sources se montrent formelles en tant que productions savantes c'est-à-dire œuvres de l'élite intellectuelle. Elles tendent ainsi à emporter la conviction de la majorité des observateurs aux prises avec des informations diffusées à travers les canaux «légitimes » (productions intellectuelles de portée scientifique). La constatation pourtant fondamentale demeure cette convergence des vues et des témoignages sur l'ambition rwandaise d'occuper une partie du territoire de la RDC. Depuis les années 1990, et particulièrement après le génocide de 1994 ayant déversé les réfugiés rwandais sur le territoire congolais, les déclarations de sources diverses n'ont cessé de faire mention de la constitution de l'Etat hima-tutsi en séparation avec les Hutu.

On ne saurait perdre de vue le fait que la globalisation s'accompagne des processus de recompositions territoriales dans beaucoup de régions du monde (Yengo, 2002). Cette réalité est bien présente en RDC où, depuis février 2007, des discussions ont été engagées entre les gouvernements angolais et congolais au sujet des frontières déclarées violées par l'Angola. Cette zone déclarée riche en diamant, le territoire de Kahemba dans la province frontalière de Bandundu (à l'Ouest), n'a-t-elle pas risqué de consacrer la crise de cohabitation sinon la rupture d’une amitié tissée hier au plus fort de la guerre de conquête impérialiste que dénoncent les milices maï-maï ?

L'idée de l'implosion par atomisation de l'Etat congolais peut être aussi lue à travers cet imaginaire populaire de l’impérialisme. Sans forcément respecter le calendrier fixé (le chronogramme), certains des éléments repris dans ce plan ont été réalisés avec succès par le RCD : création de nouveaux territoires, jadis inexistants, à dominance Tutsi ou 
Banyamulenge (Tutsi congolais) comme la localité de Kilembwe érigée en territoire dans le Sud-Kivu (Ministère des Droits humains-RDC, 2001 ; Migabo, 2004), la création des assemblées provinciales, le projet étouffé dans l'œuf d'injecter la monnaie rwandaise dans les transactions commerciales en territoire sous contrôle rebelle non approvisionné par Kinshasa (la capitale légale), le projet de constitution sans un Etat déclaré tel, le projet de l'hymne national ... autant des signes qui ne trompent pas et qui confortent la thèse impérialiste sous l'influence rwandaise dans l'Est de la RDC.

Que l'on ne perde pas de vue que même les mensonges modernes ne se passent pas de faits pour mieux se justifier. Les mensonges politiques modernes portent sur des faits, non sur des intentions, et sur des faits connus publiquement. Arendt distingue ainsi deux grands types de mensonges modernes: la réécriture de l'histoire contemporaine et la fabrication d’images (citée par Piotte, 2005, p. 580). La tendance, par exemple, de certains auteurs ou acteurs historiques à exagérer la description de certains faits ou événements, les aggraver ou les simplifier à l'excès pour les minimiser, comme ce fut avec le génocide de 1994 au Rwanda, est une pratique qui a été constatée et dénoncée par nombre de chercheurs (Newbury, 1998 ; Pottier, 2002 ; Bernault, 2004).

\section{"La réunion tenue en Amérique. Document Top secret. Processus de la guerre de conquête des Tutsi 'Bahima' "}

Ce deuxième document ne comporte aucune indication relative au lieu et à la date de son établissement. Il reprend cependant trois noms des présumés représentants du plan dit «diabolique » d’occupation du Congo : Paul Kagame (Président du Rwanda), Museveni Kaguta (Ouganda) et Bizima na Karahamuheto (sujet Munyamulenge ou Tutsi congolais selon des formules consacrées par l'usage dans la région), signant "Pour le compte du conseil de planification de la guerre ». Ce document qui comporte trente-deux (32) dispositions ou articles, fait particulièrement allusion à des actions traduisant la participation, directe ou indirecte, des puissances anglo-saxonnes aux côtés du Rwanda pour la réussite du processus en cours. On peut relever ceci :

'Dans l'avenir, on parlera de «Bahima », plus de Tutsi ou Banyamulenge pour l'Empire 'Hima' supporté par les USA' ; les pays des Grands Lacs deviendront l'empire 'Hima' plus tard et les USA placeront leur base militaire sur le territoire de l'île de Idjwi pour notre protection’ (article 28) ;

'Nous imposons la langue anglaise comme langue internationale pour nous servir d'appui aux Américains et aux Britanniques contre la francophonie’ (article 32) 
Le document parle aussi des questions relatives au pouvoir traditionnel, au rôle des détenteurs des forces mystiques et à la supériorité des guerriers maï-maï dont la crainte implique qu'on y mette fin pour la réussite du présumé plan tutsi au Congo. En voici les articles :

'Exterminer les autorités coutumières et locales pour affaiblir le pouvoir coutumier et les guérisseurs traditionnels' (article 5) ;

'Déstabiliser les territoires et villages qui entraînent la guerre populaire maï-maï car ils ont la force contre nous les Tutsi ' (Article 21).

Par rapport aux jeunes qui constituent la pépinière pour le recrutement de la main-d'œuvre et des combattants, il est dit ceci dans le document:

'Massacrer des jeunes congolais pour prison collective, exercices lourds, travaux forcés (article 5) ;

'Affaiblir la démographie du Kivu en tuant les enfants masculins, les enseignants, y compris les femmes intellectuelles, les autres femmes survivant comme esclaves sans plus être fécondées' (Article 13)

Sur les enjeux de l'exploitation minière et la guerre, on peut $\mathrm{y}$ lire :

' Les Tutsi-Hima et les étrangers alliés doivent orienter leurs activités à l'exploitation minière pour l'achat des armées ${ }^{25}$ (article 16) ;

'Exterminer tous les militaires des FAC surtout les officières et les techniciens de guerre' (article 2);

'Piller des armes et munitions de guerre vers le Rwanda et l'Ouganda pour servir des bases arrières' (article 7) ;

'Pillage des richesses du pays surtout les minerais et produits agricoles et industriels' (article 8).

Pour souligner la «haine ethnico-raciale" que les Tutsi développeraient à l'égard des Hutu (Bantous), il est dit :

'Exterminer tous les Hutu vivant au Congo en commençant par les réfugiés Hutu que les Tutsi appellent communément «Adui » (swahili du mot 'ennemi'). 'L'ennemi des siècles passés, présents et l'avenir' (article $\left.1^{\mathrm{er}}\right)$.

Le document dit aussi : 'Tuer le président en exercice et occuper le poste de la présidence du RCD. A la tête Rwakabuba et Bizimakara' (article 10).

${ }^{25}$ Erreur contenue dans le document. L'on comprend bien qu'il s'agit d'armes, et non d'armées. 
Du reste, notons que ce document comporte beaucoup bien d'autres articles reflétant l'ampleur des problèmes qui fondent les griefs des acteurs nationalistes dits Maï-Maï malgré la perspective apocalyptique qu'ils adoptent. Notamment, on ne peut ignorer le fait que le président L.-D. Kabila est mort le 16 Janvier 2001, clôturant à ce jour la liste des nationalistes établie par le 'prophète-libérateur' Kabambe (chef des Maï-Maï du Maniema): Simon Kimbangu a «créé » son église en 1921 et est mort en 1951 ; Patrice E. Lumumba est mort en 1961 et Kabila en 2001. Entre 1921, 1961 et 2001, il y a une différence symbolique et constante de 40 ans qui rappellent le long séjour des Israélites dans le désert partant de l’Egypte pharaonique (une puissance de l'époque) en vue de rompre avec la domination impérialiste (Dussel, 1985 ; Strensky, 2006 ; Gutierrez, 2006).

En dépit des écarts ou distorsions constatés entre ce qui a été prédit et ce qui est vécu, il apparaît que la plupart des articles (dispositions) présentés plus haut, correspondent à des faits réels pouvant servir de preuves dans nombre de séquences de guerres connues ces dernières années (Lhuilier, 2013). L'évolution politique récente semble avoir démenti certaines prévisions mais l'incertitude plane encore et le danger n'est pas totalement évité (Clark, 2002 ; Vircoulon, 2010).

\section{"La charte de l'impérialisme »}

Ce document ne comporte ni le lieu ni la date de son établissement. Il comprend dix-neuf dispositions regroupées en six titres. En fait, l'intelligibilité des faits relatifs au phénomène d'impérialisme qui en découle est de nature à frapper l'imagination des lecteurs et des auditeurs que forment les masses, en majorité incultes, recevant les leçons de conscientisation de la part des Maï-Maï. Il fait le tour d’horizon, à l’instar du document précédent, de tous les grands problèmes contemporains relatifs à la vie des Etats du tiers monde aussi bien sur le plan juridico-politique que sur le plan économico-financier. Le document semble avoir délibérément adopté un raisonnement procédant par antithèse pour subvertir des formules consacrées et utilisées dans les traités internationaux et les chartes régissant la vie internationale des Etats. Les concepts-clés comme la souveraineté, les droits de l'homme, la démocratie, la justice, sont ici remis en question sur la base des réalités vécues par les acteurs sociaux locaux. En fait, il est connu que les Congolais ordinaires sont confrontés au quotidien à des situations décrites comme relevant du tragique par la quasi-totalité des médias indépendants.

Pour pousser les masses à la mobilisation, on peut relever dans le document ce qui suit:

'Proclamant notre adhésion à la charte de l'impérialisme et à la

Déclaration Universelle des droits de l’impérialisme; soucieux de 
maintenir les pays du Tiers-Monde dans la misère, la division, la guerre, la confusion et l'anarchie, affirmant notre détermination politique, économique, sociale et culturelle de nos pays en vue de mieux les exploiter et le piller ;

Nous, Impérialistes du monde entier, inconscients de nos responsabilités devant Dieu, Déclarons solennellement adopter la présente charte (préambule)'; 'Aucun pays du Tiers-Monde ne constitue un Etat souverain' (article 1er) ;

'Tous les pays du Tiers-Monde sont divisibles et leurs frontières déplaçables selon notre volonté, (article 2);

'Notre devise : divisé pour régner, conquérir exploiter et le piller pour remplir nos banques et en faire les plus puissantes du monde' (article 3) ;

'Seul un gouvernement établi par nous est légal et démocratique. Tout autre gouvernement est illégal quelle que soit sa forme’ (article 6) ;

'On ne parle pas de génocide, ni de massacre là où nos intérêts sont garantis, même si le nombre de victimes est très important' (article 12) ;

'La charte relative à l'exploitation des pays du Tiers-Monde pendant la période de la guerre froide est abrogée’ (article 18).

Sans qu'il y ait besoin d'étendre le commentaire, disons simplement que la perception de la crise induite par la globalisation avec la conjoncture des guerres à visées économiques et les violences qu'elle implique, est bien nette dans les extraits de ladite Charte imaginaire. L'impact de cette dernière est cependant de nature à cristalliser la haine vis-à-vis des individus ou des groupes étrangers se réclamant attachés à quelque puissance ou à quelque organisation internationale investie des prérogatives dans les matières citées dans la Charte attribuée aux impérialistes.

Le bilan mitigé de la Mission de l'Organisation des Nations Unies en RDC (MONUC) est éloquent à cet égard : la paix déclarée solennellement comme établie à la suite des accords signés par les acteurs (ex-belligérants principalement) ne représentant pas le peuple congolais de manière légitime au niveau national, contraste avec la recrudescence des violences dans les milieux ruraux représentant le local complexe. La MONUC, remplacée aujourd'hui par la Mission de l'Organisation des Nations Unies pour la Stabilisation du Congo (MONUSCO) à la suite de la reconfiguration du mandat, reste présente dans les esprits des hommes ordinaires et que rien de visiblement matériel ne saurait persuader sur les possibles progrès en matière de sécurité sur le terrain du Congo profond qui leur sert de baromètre (Olombi, 2015 ; Vircoulon, 2005, 2007 ; Tertrais, 2016). 
Les affrontements de 2007 à Kinshasa entre la milice de l'ex-chef rebelle et ex-vice-président Jean Pierre Bemba, devenu sénateur à la faveur de la constitution de la transition, et l'armée régulière sous le regard passif de la MONUC, sont édifiants sur l'évaluation des capacités réelles, ou mieux de la volonté réelle de l'Organisation des Nations Unies (ONU) à résoudre les problèmes des pays du tiers-monde.

Très fondamentale encore, apparaît la constatation faite dans cette Charte impérialiste sur le changement radical du mode de gestion des Etats périphériques à la fin de la division binaire du monde (article 18). En effet, le monopole idéologique du bloc libéral, induit par l'effondrement de l'empire soviétique (ex-URSS), s'affirme davantage dans le domaine économique par le principe globalisant de la pensée unique. C'est la phase de l'intolérance et de la désolation, estiment nombre d'observateurs nostalgiques de la guerre froide, celle-ci étant alors perçue comme la matrice pour l'analyse des grands problèmes politiques internationaux contemporains. Si hier on comptait sur les bonnes grâces de deux blocs (l'Ouest libéral et l'Est socialiste) en raison de leurs états d'âme dictés par la logique de la concurrence et la nécessité de préserver leurs zones d'influence respectives, rétablissement de l'équilibre sur la scène internationale (French, 2005).

De ce qui précède, il y a lieu de tenter une théorisation qui conforte notre argumentation sur les stratégies de mobilisation populaire en faveur des entreprises miliciennes. En effet, la question des documents écrits produits pour alimenter, ou mieux donner corps à la rumeur, n'échappe pas aux préoccupations scientifiques en matière de communication et information. A ce sujet, Pierre-Claver Ndayicariye fait observer que «le document écrit confère la pérennité à la parole" (Ndayicariye, 1998, p. 52). Et la commission Sean Mac Bride d'ajouter : « Le développement de l'écriture a permis de préserver les messages porteurs des symboles les plus lourds de sens, garantissant ainsi l'existence permanente de la communauté. Les livres écrits et copiés à la main, ont une histoire qui remonte à trois millénaires. Par extension, le livre est devenu l'inégalable dépositaire de la pensée et de la connaissance dans la plupart des grandes civilisations des époques classiques " (Ndayicariye, p. 52). Appuyées par des documents écrits ou tracts, " les rumeurs, anonymes et cyniques, portent souvent la force de la déstabilisation des institutions, l'ambition de démolir dans l'opinion l'image des institutions, les affaiblir, c'est-à-dire diminuer leurs poids et leur autorité dans l'environnement interne et externe» (Ndayicariye, p. 52).

Poursuivant l'effort de théorisation des enjeux de la rumeur pour lesquels les documents écrits et les tracts servent de vecteurs d'authentification et de légitimation, Ndayicariye propose les principes 
d'analyse qui permettent de comprendre l'impérialisme des rumeurs et leurs répercussions sur la vie sociopolitique ${ }^{26}$.

Comme on peut s'en rendre compte, les différents principes énoncés au sujet de l'analyse des rumeurs à travers celle des documents qui les soutiennent, semblent avoir été solidement exploités par les milices maï-maï face aux Tutsi et leurs alliés congolais impliqués dans la guerre en RDC. Nous avons parlé de l'impérialisme imaginaire pour insinuer qu'il s'agissait, comme encore aujourd'hui, d'une rumeur alimentée par la production des documents écrits devant servir des supports réels et crédibles à la stimulation des masses à la lutte contre une réalité impérialiste et ancienne. Toutefois, il est pertinent de relever le danger de cette instrumentalisation de l'écrit en tant que forme de mensonge politique moderne. Selon Arendt,

"Ces mensonges organisés peuvent conduire les trompeurs à se duper eux-mêmes, à croire à l'image fabriquée et à perdre tout contact avec la réalité. Ils peuvent mener à la violence dans la mesure où ils portent à détruire ce qu'ils nient, comme l'a montré l'expérience des gouvernements totalitaires. Ils conduisent le plus souvent au cynisme, c'est-à-dire à l'incapacité de s'orienter à travers les informations et les images véhiculées. » (Citée par Piotte, 2005, p. 580).

\section{Conclusion}

L'examen fait de documents relatifs à la mise en œuvre de la stratégie de dénonciation de l'impérialisme par les milices maï-maï du Maniema a permis de mettre en relief la capacité mobilisatrice incarnée par les écrits dans toute société moderne. A fortiori, lorsqu'une telle stratégie est déployée par une élite dans un espace dominé par des acteurs sociaux incultes, l’on

${ }^{26}$ D’après l'auteur, ces principes s’énoncent comme suit : « -On ne doit pas remonter à l' origine, au « concepteur » des rumeurs. Les « laboratoires » prennent toutes les précautions pour réduire le risque. Tous les coups sont permis et plusieurs relais utilisés. La filière apparente n'est pas souvent la filière réelle. Ce qui complique, pour les cibles, la riposte ; Les buts d'une rumeur ne sont pas toujours apparents. Il est parfois difficile de savoir qui est réellement visé par la rumeur. Le degré du fictif et de l’incertain augmente dans le jeu politique et aggrave le trouble de l’opinion ; -Pour réussir, l’opération suppose une certaine préparation psychologique, une certaine sensibilisation. Dans ce cas, la phase de préparation, de mise en condition de l’opinion, ou de désinformation est nécessaire pour créer après l'événement. Cette phase, difficile à saisir appartient au clandestin ; -Les médias jouent un rôle essentiel dans l'exploitation politique des rumeurs. Les médias ont une force d'amplification, ils ont la capacité de donner forme et profondeur a la rumeur ; -Il y a des rumeurs qui créent un événement politique. Il y en a d'autres qui ont une vie éphémère et qui ne franchissent pas la barre ; -Le but d'une rumeur est un changement de rapports de force quel que soit le coût. C’est pourquoi une rumeur est considérée comme une opération de guerre à faibles coûts. Opération de guerre parce que il y a toujours intérêt à créer le trouble chez l'adversaire ». Ibidem, p. 53-54. 
voit bien comment peut s'intensifier l'impact du discours, surtout dans un contexte où les destinataires ont la possibilité de faire valoir leur situation de victimes.

En outre, l'analyse permet de comprendre dans le contexte de la guerre du RCD jouissant d'un soutien sans faille de ses alliés rwandais, comment plusieurs processus se sont combinés en fonction de catégories d’acteurs. Ainsi, les milices maï-maï manifestement marquées par une élite guerrière, ont contribué à la politisation à outrance de masses rurales par la diffusion des discours à caractère politique et idéologique autour des notions de pouvoir illégitime, de domination illégitime, de nationalisme, d’impérialisme, de souveraineté, de terres ancestrales (le sol et sous-sol), d'autorité traditionnelle... Leur ambition à court terme consistait pourtant à persuader les populations du bien-fondé de leur lutte pour faciliter le recrutement de nouveaux combattants et de renforcer l'hostilité à l'égard des rebelles et leurs alliés.

Autant de questions essentielles touchant à la vie des communautés rurales sous le double impact des rebelles et des milices. Ces communautés constituaient pour les milices une base des ressources diverses dont elles avaient besoin pour faire face à l'ennemi (Amuri, 2008b). Cette socialisation politique qui s'est opérée d'une façon quelque peu diffuse, a à la fois induit la politisation et généré le réflexe contestataire des populations rurales, non seulement vis-à-vis des rebelles et leurs alliés rwandais, mais également à l'égard des combattants maï-maï eux-mêmes, dont les abus ont fini par faire tomber le masque nationaliste. La transition post-conflit de 2003 à 2006 ainsi que les élections qui ont conduit à la mise en place des institutions de la Troisième République, ont permis d'étaler au grand jour la part, non moins significative, de la crise de légitimité de la plupart des candidats d’obédience maï-maï, sanctionnés lors des élections de 2006 et 2011 par les populations de leurs terroirs respectifs. De ce point de vue, la démocratisation apparaît bien un vecteur puissant de la politisation des acteurs ruraux.

En termes de prospective politique, soulignons que l'activisme poussé des jeunes, qui s’affirment aujourd'hui dans les grandes villes au nom de la démocratie, de la bonne gouvernance et des droits de l'homme, semble prolonger la tradition forgée dans les enclaves rurales sous l'impact de l'idéologie d'un nationalisme fanatique pendant les années de guerre. La persistance des pratiques autoritaires est loin d'augurer des lendemains meilleurs, notamment l'alternance politique pacifique qui était attendue en 2016 (Gerold et Sullivan, 2017). De plus en plus, les manifestations populaires mettant en évidence le caractère contestable et contesté de l'actuel Etat congolais (entendez, le régime en place) tendent à se généraliser. Cellesci vont de l'espace rural à celui urbain sur fond de violences, de l'Est 
profond (le plus médiatisé depuis les années 90) à l’Ouest, en particulier la capitale Kinshasa et la province du Bas-Congo avec l'énigmatique mouvement politico-mystico-religieux Bundu dia Kongo ${ }^{27}$, BDK, ou sa nouvelle version Bundu dia Mayala, BDM, du chef politique et spirituel Ne Mwanda Nsemi, en passant par le centre, notamment le Kasaï Central avec le phénomène Kamuina $\mathrm{Nsapu}^{28}$. Globalement, les faits analysés sont significatifs quant à l'impact idéologique exercé sur les acteurs locaux situés dans un contexte d'instabilité structurelle des deux dernières décennies malgré l'accompagnement de la communauté internationale.

\section{Remerciements}

L'auteur, ASP alumnus, remercie vivement tous les membres du consortium Five College African Scholars Program de Massachusetts (Amherst), en général, et les professeurs David Newbury, Catharine Newbury et John Higginson, en particulier, de leurs diverses contributions à sa recherche lors de son séjour à Amherst (janvier-mai 2007) dans le programme d'études africaines.

\section{References :}

1. Adam, C. (1999). “Maji Mulele Maji”. Et les fusils ne crachent que de l'eau, Beersel (Belgique), Clepsydre.

2. Amuri Misako, F. (2012). La symbolique de la légitimation de la violence milicienne en Afrique. Continuités et réinventions $d u$ messianisme nationaliste chez les Mä̈-Maï du Maniema au CongoKinshasa, Sarrebruck, Editions universitaires européennes.

3. Amuri Misako, F. (2008a). Les milices maï-maï au Maniema (Aout 1998- Juin 2003) : un mode d'affirmation politique des masses rurales ? Mémoire de DES (inédit) en SPA, FSSAP, Université de Kisangani.

4. Amuri Misako, F. (2008b). « Les milices congolaises et la rhétorique religieuse chrétienne dans la conquête de l'espace public: A propos des milices maï-maï du Maniema (Congo-Kinshasa) », Panel on DRC, 12th General Assembly of CODESRIA, Yaounde-Cameroon, December 7-11.

\footnotetext{
${ }^{27}$ Populaire dans les milieux kongo, ce mouvement est bien connu pour ses velléités irrédentistes, prétendant en ce $21^{\mathrm{e}}$ siècle lutter pour la restauration d'un royaume Kongo disparu pendant la formation de l’Etat Indépendant du Congo (EIC) du roi Léopold II, né du partage de l'Afrique par les puissances occidentales européennes lors de la Conférence de Berlin (1885).

${ }^{28}$ Une milice associée au pouvoir traditionnel. Sans surprise, le chef assassiné s’appelait Kamuina Nsapu. Ce nom signifierait « fourmi noire » (« Black Ant »). Lire: The Economist, «Like father, like son: Why Joseph Kabila’s ambition endangers Congo. Congo's president clings to power even as the hinterland erupts », June 3rd, 2017.
} 
5. Amuri Misako, F. (2007). «De la ‘démocratisation’ de la violence à la 'milicianisation' des jeunes: (re)découvrir la rationalité du désordre », dans Maindo Monga Ngonga, A. (coord.). (2007). Des conflits locaux à la guerre régionale en Afrique centrale. Le CongoKinshasa oriental 1996-2007, collection Archive congolaise, Paris, L’Harmattan, pp. 47-60.

6. Amuri Misako, F. (2004). « Espace démocratique et violence au Congo-Zaïre : à propos du phénomène maï-maï », Paper presented at the 30th Anniversary Conference of the African Association of Political Science (AAPS) on the theme " Conflicts and African Renaissance », Yaounde-Cameroon, June 28-30.

7. Arrêté Départemental nº $001 \mathrm{MJ} /$ Dat/route/1999 portant création à titre provisoire de Territoire de Bunyakiri, Minembwe et Kasha au Sud-Kivu.

8. Banégas, R. (2008). «La République oublie-t-elle ses enfants ? » Milicianisation et démilicianisation du champ politique en Côte d'Ivoire », communication donnée au colloque Regards croisés sur les milices d'Afrique et d'Amérique latine en situation de violence, CERI, du 25 au 26 septembre.

9. Benhammou, M. (2017). Le djihadisme international : l'ennemi invisible. Mutations idéologiques et stratégies opérationnelles, collection Diplomatie et Stratégie, Paris, L’Harmattan.

10. Bernault, F. (coord.). (2004). «L’écriture scientifique en temps de crise ", Afrique et Histoire, no. 2.

11. Brochard, F. (2016). Boko Haram? Pourquoi pas. C'est mieux que mourir en prison, non? Chroniques de la prison de Bafia, 2010-2014, Paris, L'Harmattan.

12. Clark, J.F. (2002). The African Stakes of the Congo War, MacMillan, Palgrave.

13. Collier, D. et Levitsky, S. (1997). “Democracy with Adjectives: Conceptual innovation in Comparative Research”, World Politics, Vol, 49, n³, pp. 430-451.

14. De Villers, G. (2016). Histoire du politique au Congo-Kinshasa : les concepts à l'épreuve, Louvain-la-Neuve, Academia-L’ Harmattan.

15. Diamond, L. (2002). “Thinking about Hybrid Regime”, Journal of Democracy, Vol 13, n 02, pp. 21-36.

16. Dussel, E. (1985). Philosophy of Liberation, Oregon, Orbis Books.

17. French, H. W. (2005). A Continent for the Taking: the Tragedy and Hope of Africa, New York: First Vintage Books editions.

18. Gerold, G. et Sullivan, Th. (2017). “République démocratique du Congo: une alternance pacifique est-elle encore possible?”, NoteFondation pour la Recherche Stratégique, no 04/17 du 16 février. 
19. Gutierrez, A. (2006). Theology of Liberation, New York, Orbis Books.

20. Koffi, B.-F. (1993). Le pouvoir de la brousse. Ni démocratie ni développement en Afrique noire sans les paysans organisés, Paris, L'Harmattan.

21. Ledda, R. (1967). “ Les classes sociales et lutte politique” Revue internationale du socialisme, $\mathrm{n}^{\circ} 22$, août, pp. 594-615.

22. Lhuilier, G. (2013). « Minerais de guerre. Une nouvelle théorie de la mondialisation du droit", Fondation Maison des Sciences de l'homme-Working Papers Series (FMSH-WP), 36, juillet, 41 pages.

23. Lovens, M. (1974). "La révolte de Masisi-Lubutu (Congo belge, janvier-mai 1944) ", in Cahiers du CEDAF, No 3-4, première partie, Série 2 : Histoire.

24. Lubala Mugisho, E. (2000). «L'émergence d'un phénomène résistant au Sud-Kivu» Afrique des Grands Lacs 1996-2000, pp. 193-228.

25. Migabo Nshurani, V. (2004). «Les motivations de l'érection de certaines circonscriptions administratives en territoires dans la province du Sud-Kivu», Revue de l'IRSA, n¹0, décembre, pp. 41-53.

26. Ministère des Droits humains de RDC. (2001). Livre blanc. La guerre d'agression en République Démocratique du Congo : Trois ans de massacres et de génocide "à huis clos », numéro spécial, Kinshasa, octobre.

27. Mulambu Mvuluya, F. (1970). «Introduction à l'étude du rôle des paysans dans les changements politiques», Cahiers économiques et sociaux, IRES, Vol VIII, n³, septembre, pp. 435-450.

28. Mutuza Kabe, R.-E. (2004). La problématique du mythe hima-tutsi, Kinshasa, Noraf.

29. Mwene-Batende, (1982). Mouvements messianiques et protestation sociale. Le cas du Kitawala chez les Kumu du Zaïre, Kinshasa, Faculté de Théologie catholique,

30. Ndayicariye, P.-C. (1998). " Le document au service de la rumeur », communication présentée au Colloque international sur Rumeurs de paix, sociétés en convalescence et liberté d'information, organisé par le Réseau Documentaire international sur la région des Grands Lacs Africains à l'Université du Burundi du 07 au 09 décembre.

31. Newbury, C. (2002). "States at War: Confronting Conflict in Africa», African Studies Review, Vol. 45, No. 1, April, pp. 1-20.

32. Newbury, D. (1998). "Understanding Genocide", African Studies Review, Vol. 41, No. 1, April, pp. 73-97.

33. N'sanda Buleli Tchungu. (2004). «La culture swahilie et l'occupation des traitants arabo-swahili dans le Maniema à l'Est du Congo-Zaïre 
au milieu du XIXeS», in Les Cahiers de l'ISP Kindu, no 004, septembre, pp. 21-30.

34. N’Sanda Buleli, L. (2003a). «La bataille de Kindu ou la leçon d'une défaite », Cahiers Africains, no 60.

35. N’Sanda Buleli Tchungu. (2003b). La mémoire et l'histoire dans les constructions politiques régionales et ethniques au Maniema pendant la transition (1990-2000), Thèse de doctorat en histoire, Faculté des études supérieures, Université Laval (Québec), octobre.

36. N’Sanda Buleli, L. (2001). «Le Maniema, de la guerre de l'AFDL à la guerre du RCD », Politique africaine, no 84, décembre, pp. 59-75.

37. Olombi, J.-C. (2015). Guerre et paix : leçons de l'intervention de l'ONU en République Démocratique du Congo, collection Etudes africaines, série Politique, Paris, L’Harmattan.

38. Piotte, J.-M. (2005). Les grands penseurs du monde occidental. L'éthique et la politique de Platon à nos jours, $3^{\mathrm{e}}$ édition, Québec, Fides.

39. Pottier, J. (2002). Re-Imagining Rwanda: Conflict, Survival and Disinformation in the Late Twentieth Century. Cambridge: Cambridge University Press.

40. Salmon, P. (1994). Processus Démocratique en Afrique : Impact et perspectives, Belgique, ULB/CERI.

41. Tertrais, B. (2016). «Les interventions militaires, cause de terrorisme? », Note-Fondation pour la Recherche Stratégique, no 06/16 du 15 février.

42. Thiriot, C. (2000). Trajectoires de libération en Afrique contemporaine (Hommage à Robert Buijtenhuijs), Paris-Leyde, Karthala-ASC.

43. Van De Walle, N. (2002). «Africa's Range of Regimes”, Journal of Democracy, n¹3, pp. 66-80.

44. Verhaegen, B. (1969). Rébellions au Congo, tome II. Maniema, Bruxelles, CRISP.

45. Vircoulon, Th. (2010). «Autopsie d'une controverse internationale. Le partenariat sino-congolais sous le feu des critiques », Les Temps Modernes, $1 \mathrm{n}^{\circ}$ 657, pp. 73-81. DOI : 10.3917/ltm.657.0073

46. Vircoulon, Th. (2007). «L'Etat internationalisé. Nouvelle figure de la mondialisation en Afrique », Études, 1 (Tome 406), pp. 9-20.

47. Vircoulon, Th. (2005). «Ambiguïtés de l'intervention internationale en République démocratique du Congo », Politique africaine, 2 ( $\mathrm{N}^{\circ}$ 98), pp. 79-95. DOI 10.3917/polaf.098.0079

48. Yengo, P. (2002). "Mondialisation, nouvel ordre de la guerre et permanence des conflits en Afrique”, Bulletin du CODESRIA, n ${ }^{\circ} 3$ et 4, pp. 55-65. 